\title{
Behavioral contrast and reinforcement value
}

\author{
BEN A. WILLIAMS \\ University of California, San Diego, La Jolla, California
}

\begin{abstract}
Behavioral contrast was produced in two target components of a four-component multiple schedule by having two target stimuli followed either by a higher rate of reinforcement or by extinction. Response rate was higher in the target followed by extinction. Periodic probe trials were then presented in which the two target stimuli were presented together. Choice on these probe trials was in favor of the stimulus followed by the higher rate of reinforcement during regular training. Experiment 2 replicated this finding but with probe trials presented throughout training. Here, preference for the stimulus followed by the higher rate of reinforcement was evident early in training, substantially before the contrast effects developed. The results challenge interpretations of contrast based on the concept of relative value.
\end{abstract}

Since the first systematic investigation of behavioral contrast by Reynolds (1961), the dominant interpretation has been that contrast effects are a function of the relative rate of reinforcement (for reviews, see Freeman, 1971; Williams, 1983). That is, response rate in one component of a multiple schedule is an inverse function of the reinforcement rate in the alternative components. Such control by the "context of reinforcement" has been described quantitatively with some precision (e.g., Williams $\&$ Wixted, 1986), but the mechanisms underlying it have not been specified beyond the simple description of the quantitative relationship. The most natural interpretation of this relationship is in terms of relative value: the value of the reinforcement rate during the unchanged component of the schedule is an inverse function of the rates of reinforcement available during the alternative components of the multiple schedule. This interpretation has been made explicitly by Bloomfield (1969), who argued that "contrast is the result of elation at, or exaggeration of the value of reward in a discrimination situation which is caused by worse conditions (absence of reward) in S-." Such an interpretation is consistent with "adaptation level" explanations of contrast effects with other procedures, such as the elation and depression effects obtained with runway procedures (see Flaherty, 1982, for a review).

Although the "relative value" interpretation of contrast has considerable intuitive appeal, there is a major conceptual leap from the empirical demonstration that relative rate of reinforcement is the variable controlling the occurrence of contrast to the interpretation that relative rate of reinforcement exerts its effect by changing the perceived value of a given rate of reinforcement by altering its context. The only direct evidence in its favor comes from a briefly reported study by Bloomfield (1969). He

This research was supported by NIMH Research Grant MH 42197 and NSF Grant BNS 9010323 to the University of California, San Diego. The author expresses his appreciation to Bertram Ploog for his conduct of the experimental protocols. Reprint requests should be addressed to the author, Department of Psychology, UCSD, La Jolla, CA 92093-0109. addressed the issue by presenting pigeons two different stimuli, associated with identical reinforcement schedules, but differing in whether they were alternated with a discriminated period of extinction. Stimulus A was alternated with extinction over 2 -min cycles during 10 -min blocks, and Stimulus B was presented continuously (at different times in the same session) for 10-min blocks. Higher response rates occurred to Stimulus A, indicating that contrast had occurred. Next, both Stimulus A and Stimulus B were presented together during preference tests (the details of the test are unclear); again, Stimulus A was preferred. Bloomfield thus concluded that contrast occurred, because the subject valued the food more during the stimulus alternated with extinction.

However, other evidence is contrary to Bloomfield's interpretation. Nevin, Smith, and Roberts (1987) presented pigeons a four-component multiple schedule in which two components had identical schedules but were followed by either a higher valued schedule or a lower valued schedule. Consistent with previous results that different following schedules are a major source of contrast (Williams, 1979, 1981; Williams \& Wixted, 1986), response rate was lower in the target component followed by the higher valued schedule. They then used a "resistance-to-change" assessment of the stimuli associated with contrast, both by prefeeding the subjects prior to the session and by presentation of several consecutive extinction sessions. The result was that greater resistance to change occurred for the behavior during the stimulus followed by the higher valued schedule, an effect in the opposite direction of that obtained with response rate during the previous training. Similar results were obtained by Tota (1990). Consequently, Nevin et al. concluded that contrast was an operant performance effect and it did not correspond to the value of the stimulus itself.

There are two apparent important differences between the Bloomfield (1969) and Nevin et al. (1987) studies. The first is the manner in which contrast was produced. Bloomfield alternated one target component with extinction but not the other, whereas Nevin et al. used different fol- 
lowing schedules. It is possible that effects due to the following schedule have functional characteristics different from those of contrast effects from other sources (e.g., variation in the preceding schedule). However, it should be noted that the great majority of steady-state contrast has been shown to be due to the following-schedule effect (Williams \& Wixted, 1986). The second important difference is that the two measures of stimulus value, preference tests for Bloomfield and resistance to change for Nevin et al., are not necessarily equivalent measures. Considerable prior research has demonstrated that resistance to change and preference are highly correlated measures that respond similarly to many different manipulations that intuitively should produce variations in "response strength" (see Nevin, 1979, for a review), but it is possible that this correspondence does not hold for all variables. Thus, resistance-to-change and preference tests may depend upon different processes.

The present study was conducted to resolve the conflict between the previous studies and to provide a further investigation of the effect of behavioral contrast on stimulus value. Pigeons were trained in a four-component multiple schedule, such as that used by Nevin et al. (1987), until clear anticipatory contrast effects were obtained. Periodic probe trials were then inserted into the regular contrast procedure such that the two target stimuli were pitted against each other in a preference test. In the second phase, training was continued on a four-component schedule, but with the two target components having different preceding schedules. Again, the probe preference tests were inserted after behavior had stabilized. The issue was how the outcome of the preference tests would correspond to the response rates maintained during the regular training procedure.

\section{EXPERIMENT 1}

\section{Method}

\section{Subjects}

Four experienced White Carneau pigeons were maintained at $80 \%$ of their free-feeding body weights by additional feeding, when necessary, after each experimental session. Immediately prior to the start of this experiment, all had been trained on an anticipatory contrast procedure identical to that reported by Williams (1990a).

\section{Apparatus}

Four identical three-key operant chambers were used. Their internal dimensions were $36 \mathrm{~cm}$ wide $\times 32 \mathrm{~cm}$ long $\times 35 \mathrm{~cm}$ high. All walls were opaque gray plastic except the front, which was sheet aluminum. Mounted on the front sheet were three translucent response keys $(2.5 \mathrm{~cm}$ in diameter) located $26 \mathrm{~cm}$ above the floor and $7.25 \mathrm{~cm}$ apart, center to center. Each key required a force of approximately $0.15 \mathrm{~N}$ to operate and could be transilluminated from the rear by standard IEE $28-\mathrm{V} 12$-stimulus in-line projectors. A 28-V 1-W miniature lamp was located $8.75 \mathrm{~cm}$ above each response key; the rightmost of these lamps provided general chamber illumination. Directly below the center key and $9.5 \mathrm{~cm}$ above the floor was a $5.7 \times 5 \mathrm{~cm}$ opening that provided access to a solenoid-operated grain hopper. When activated, the hopper was illuminated from above by a $28-\mathrm{V}$ 1-W miniature lamp. Reinforcement consisted of 3-sec access to milo during which all keylights and houselights were dark. A 5-cm speaker was mounted above the center of the ceiling and provided continuous white noise throughout the experimental sessions. The experimental events in each chamber were controlled by microcomputers located in an adjacent room.

\section{Procedure}

Different following schedules. Because all subjects had prior experience, they were immediately begun on the four-component schedule used during Phase 1 . The four components were arranged in pairs, such that Component $A$ was always followed by Component $B$, Component $C$ was always followed by Component $D$, and the A-B and C-D pairs were randomly alternated. Throughout training, Components $A$ and $C$ were $10 \mathrm{sec}$ in duration, and Components $B$ and $D$ were $30 \mathrm{sec}$ in duration. These values were employed because previous work has shown them to optimize the occurrence of contrast (Williams, 1989). The schedules of reinforcement in Components $A$ and $C$ were both VI 2-min, the schedule during Component B was VI $30-\mathrm{sec}$, and the schedule during Component D was extinction.

The stimuli correlated with Components $A$ and $C$ were red and green keylights, which transilluminated the entire response key. Assignment of the colors to component was counterbalanced across subjects. These colors appeared randomly on the left and right response keys of the chamber, only one of which was illuminated at any given time. The stimulus for Component $B$ was always a white vertical line on a dark background; the stimulus for Component $D$ was always a white horizontal line. Both line orientations appeared on the center key.

Experimental sessions continued for $56 \mathrm{~min}$, which on average included 42 presentations of each component. Training in Phase 1 continued for 20 sessions. During the last five sessions, probe testing was begun, which involved simultaneous presentation of the red and green colors correlated with Components $A$ and $C$. The location of the colors on the left and right keys was randomly varied, with the constraint that each color occur in both positions an equal number of times over the course of the session. Probe trials were presented for the same $10-\mathrm{sec}$ durations as the regular presentations of Components $\mathrm{A}$ and $\mathrm{C}$ during the regular training, and their reinforcement schedules (both VI 2-min) continued to operate as well. Eight probe trials were given during each of the last five sessions-four following the presentation of Component B and four following the presentation of Component D. Probe trials were followed randomly by presentation of Component $\mathrm{A}$ or $\mathrm{C}$. The training procedure during the remaining portion of the probe sessions was the same as that during the regular training.

After the completion of the initial phase of training, the stimuli correlated with Components $\mathrm{A}$ and $\mathrm{C}$ were reversed for individual subjects. A total of 25 sessions were given with the reversed stimuli, again with probe trials occurring during the last five sessions. Except for the stimulus reversal, the procedure was identical.

Different preceding schedules. The procedure was then changed to one in which Components $A$ and $C$ were now preceded consistently by Components $B$ and $D$, rather than followed by them. The components were again arranged in pairs, such that B-A and D-C pairs were randomly interspersed across training, so that $A$ was always preceded by $B$ and $C$ by $D$, but with the components following $\mathrm{A}$ and $\mathrm{C}$ randomly determined. The stimulus assignments for Components $A$ and $C$ remained the same as during the immediately preceding training. A total of 30 sessions were presented, the last 5 of which included probe trials. The probe procedure was identical to that used in the previous phase of training.

During the last stage of training, the stimuli assigned to Components $A$ and $C$ were again reversed. In addition, the stimulus correlated with extinction (Component $D$ ) was changed from a horizontal line to a line $30^{\circ}$ from vertical, in order to increase the difficulty 
of discriminating Components B and D. A second change was that sessions with probe trials now occurred every fifth session throughout training, rather than for five consecutive sessions at the end of training. Training continued for $\mathbf{3 0}$ sessions.

\section{Results}

\section{Different Following Schedules}

Figure 1 shows the mean response rates for Components $A$ and $C$ during the initial phase of training in which they were followed by different schedules (A by VI 30$\mathrm{sec}, \mathrm{C}$ by extinction) and then after the stimuli correlated with $A$ and $C$ were reversed. Response rates for Components $B$ and $D$ are not shown because they are uninformative: response rates for Component $B$ were high for all subjects and those for Component $D$ were near zero throughout training. Because all subjects had experience with the schedule immediately prior to beginning this phase, the response rates in Component $\mathrm{C}$, followed by extinction, were substantially higher during the first block of training, and they remained so throughout the first phase of training. With the stimulus reversal, the initial pattern of response rate to the two stimuli continued for the first few sessions but then quickly reversed to where the higher rate again occurred to the stimulus followed by extinction. The results thus confirm the generality of the anticipatory contrast effect reported in numerous previous studies (e.g., Williams, 1981).

Figure 2 shows the results from the sessions with the probe trials, taken from the last five sessions of both phases of training. Thus, any effect of stimulus preference for reasons other than their associated schedules should be canceled by the reversal in stimulus assignment that occurred across the two phases of training. This averaging was necessary because there was a substantial preference for the green color independent of the schedule conditions for 2 of the 4 subjects. The right side of Figure 2 shows the data from the components when they were part of the normal schedule sequence (i.e., when Components $\mathrm{A}$ and $\mathrm{C}$ were presented individually and were followed by Components $B$ and $D$, respectively).

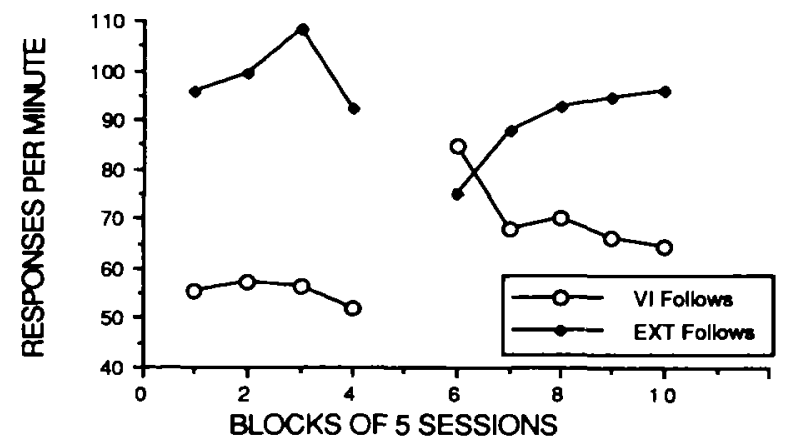

Figure 1. Response rates averaged over 4 subjects for the two target components ( $A$ and $C$ ) of the four-component schedule. The stimuli assigned to the components for individual subjects were reversed between the two phases of training.

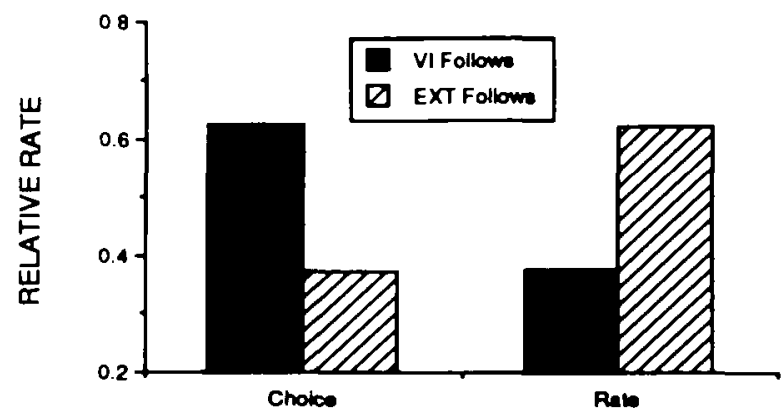

Figure 2. Results from the probe seadions expressed in terms of the relative rate of reaponding $[A /(A+C)]$. The rate mengares refer to the responses during the regular training procedure; the choice measures refer to the responses on probe trials.

The data shown thus correspond to the last data point of each line segment seen in Figure 1; here, however, they are expressed as relative rates of responding in Component $A$ versus Component $C$, rather than in terms of $a b-$ solute rates. The same relation is evident, as the higher response rate occurred in Component $\mathbf{C}$, which was followed by extinction. This difference occurred for all 4 subjects and, as assessed by a simple $t$ test, was significant statistically $[t(3)=6.05, p<.05]$.

The left portion shows the behavior when the red and green colors were presented simultaneously on the probe trials. Again, these data are averaged over both phases of training, so that any effect of stimulus preference should be canceled. Here, the opposite pattern is seen from that for the response rates when each stimulus was presented in isolation. That is, a higher rate of responding occurred to the stimulus followed by the VI component during training. This difference was also consistent for all 4 subjects (individual preference values of $0.73,0.59,0.56$, and $0.67)$ and statistically significant $[t(3)=3.47, p<.05]$.

\section{Different Preceding Schedules}

The shift to the schedule sequence in which the two target components were preceded by different schedules, and followed equally often by the same schedules, abolished the response-rate difference seen in Figure 1 during the first two or three sessions. The mean response rates remained similar throughout the first phase of training. Moreover, there was no significant difference in the rates during the two target components of the second phase, after the change in the discriminative stimuli to make them more similar. Probe tests during both phases produced a similar outcome: mean preference values approximating 0.50 in both phases. Because the failure to obtain a contrast effect due to the preceding schedule makes these preference tests uninformative, these data are not presented in detail.

\section{Discussion}

The effects of the following schedule of reinforcement were the same as those obtained in previous studies: robust 
anticipatory contrast effects occurred in the target components preceding the different following schedules, such that higher response rates occurred when the target component was followed by extinction than when it was followed by a higher valued schedule (cf. Williams, 1979, 1981). Despite these effects on response rate, choice between the two stimuli associated with the two target components was in favor of the stimulus followed by the higher valued schedule. Thus, response rate produced an effect in one direction, whereas choice between stimuli produced an effect in the opposite direction. It should be noted that the conditions on the choice probe trials were identical to those during the regular training procedure except for the presence of the alternative stimulus; the same stimulus durations were used, and the reinforcement schedules for each stimulus remained in effect. Despite this similarity, the rate and preference measures were inversely related. The apparent implication is that the allocation of responding to an alternative and the response rate to that alternative presented alone are under the control of different variables. Such a dissociation between the choice and rate measures is unusual, but not unprecedented (cf. Pearce \& Collins, 1987; Picker \& Poling, 1982).

A possible reason for the dissociation between preference and response rate seen in the present experiment is that the subjects discriminated the probe trials as having different reinforcement contingencies. Perhaps they learned that the probe trials were followed equally often by the same events, thus preventing the usual dynamics of anticipatory contrast from operating. The difficulty with this explanation is that it would apply only if the probe trials had yielded a preference near indifference. There is no obvious reason why such discrimination should produce an effect in the opposite direction. It also should be noted that the opportunity for such discrimination was minimized by presenting the probe trials very infrequently. Further evidence against the discrimination idea is that there was no systematic change in the outcome of the probe tests over the consecutive test sessions.

The dissociation between response rate and preference seen in the present experiment is consistent with the results of Nevin et al. (1987), who used a resistance-to-change measure. In their study, response rate was also greater in the target component followed by the lower valued schedule, whereas resistance to change was greater in the target component followed by the higher valued schedule. Thus, the present findings are consistent with previous results in showing that resistance to change and preference covary as a function of the reinforcement parameters.

Taken together with the results of Nevin et al. (1987), the present findings suggest that behavioral contrast cannot be taken as evidence that the unchanged reinforcement schedule (and its correlated stimulus) has an increased "value" as a result of the lower reinforcement rates in the surrounding components of the schedule. Preference, rather than response rate, has been the standard assay for determining value, as indicated by preference being a more orderly function of the reinforcement parameters that intuitively determine reinforcement value. For example, in autoshaping procedures higher response rates occur with smaller percentages of reinforcement, but preference is monotonically related to percentage of reinforcement (Picker \& Poling, 1982). Similarly, for simple reinforcement schedules, VR schedules routinely produce higher rates than do VI schedules when both are presented in isolation, but choice between them is determined by the relative reinforcement rates associated with the two schedules (Herrnstein \& Heyman, 1979). Given the precedence of choice over rate measures in other comparisons, the apparent implication is that contrast effects are associated with a decreased value of the unchanged component, rather than an increased value. Thus, the present results sponsor a conclusion exactly opposite to that of Bloomfield (1969). Why his choice procedure produced an effect opposite to that obtained here is uncertain and cannot be ascertained because of the sketchiness of the report of his results.

A critical issue raised by the present results is their generality for other contrast procedures. Contrast due to the following schedule of reinforcement has the peculiar property of producing an effect on response rate that is in the opposite direction of the Pavlovian signaling properties embedded in the procedure. That is, the target component signaling the higher valued following component should possess some of the properties of a Pavlovian CS + , so it should be expected to engender responding due to the Pavlovian contingency. In fact, autoshaping to a CS + that signals the availability of reinforcement in a subsequent component has been directly demonstrated by Brown, Hemmes, Coleman, Hassin, and Goldhammer (1982). In contrast procedures, however, this Pavlovian effect is in competition with dynamics of contrast, as shown by Williams (1979, Experiments 2 and 3) and Williams (1990a). In those studies, the Pavlovian effect was evident when there were no differential signals correlated with the different following schedules, thus causing the target stimuli to be the only sources of information about the following schedules. This was evidenced by a higher rate to the stimulus followed by the higher valued following schedule, although this effect did not occur for all subjects. However, when the differential stimuli were available, the increased response rate caused by the Pavlovian contingency was replaced by the opposite effect-namely, a higher response rate in the component followed by extinction. Such results show that the interaction between contrast and the Pavlovian contingency may be complex and raise the question of how the Pavlovian contingency was involved in producing the preference results found here.

In principle, it should be a simple matter to determine whether the inverse relation between the preference and response rate measures would hold for contrast effects due to variables other than different following schedules. In Experiment 1, we attempted such an analysis by having the different target stimuli preceded by different schedules rather than followed by different schedules. No evidence of any differential effect on stimulus preference as 
a function of the different preceding schedules was evident, but the results were ambiguous because there were no reliable contrast effects. Similar failures to show consistent effects of the preceding schedule have been observed in previous studies (Williams, 1979, 1981; Williams \& Wixted, 1986).

To further explore the circumstances under which the inverse relation between contrast and stimulus preference occurs, Experiment 2 was conducted in an attempt to produce more robust contrast effects due to the preceding schedule. The more difficult discrimination between Components B and D (vertical vs. oblique lines) was used from the start of training, in the hope that the more difficult discrimination might produce contrast more readily. In the second stage of the experiment, the sequence of components was changed to one in which the two target stimuli were followed by the different schedules, rather than preceded by them. Unlike Experiment 1, the preference tests in Experiment 2 were interspersed throughout training rather than presented only at the end of training, in order to determine how the development of the two effects were related. To the extent that they are under the control of the same variable, their development should be correlated. However, if their development is not correlated, this would be evidence that the rate effects and preference effects are due to different processes.

\section{EXPERIMENT 2}

Method
Subjects and Apparatus
Four new White Carneau pigeons were maintained as noted in
Experiment 1. These subjects had experience with a discrete-trial
conditional discrimination procedure. but with different stimuli from
those used here. The apparatus was the same as in Experiment 1 .

\section{Procedure}

The multiple schedule was the same as that used in Experiment 1. During Phase 1 , the four components were arranged in pairs such that Component $B$ always preceded Component $A$, and Component $D$ always preceded Component $C$. These $B-A$ and $D-C$ pairs were then randomly varied with respect to order of presentation. Training with these schedules continued for 30 sessions, each $56 \mathrm{~min}$ in duration. In addition to these regular sessions, a session with probe trials was presented after every block of five regular sessions. These probe sessions were exactly like those in Experiment 1, with 8 different probes occurring during each probe session-four following a B-A pair and four following a D-C pair. The regular schedule of reinforcement associated with each target stimulus (VI 2-min) was available on probe trials. The regular sequence was resumed after probe trials, with the next component being either Component $B$ or $D$, randomly determined.

During Phase 2, the sequence of components was changed to one in which the target stimuli were consistently followed by the different schedules. Thus, A-B and C-D pairs were randomly interspersed. Again, 30 sessions were presented and, after every five regular sessions, a probe session identical to that used in Phase 1 was presented.

\section{Results}

As in Experiment 1, no reliable difference was obtained in the target components as a function of the different preceding schedules. During the last 10 sessions, the mean response rate during the component preceded by extinction was 51.7 responses/minute, and that during the component preceded by the VI 30 was 47.4 responses/minute; this difference did not approach statistical significance $(F<1)$. The outcome of the probe trials was similar: the average preference for the component preceded by the VI 30 -sec schedule was $\mathbf{0 . 5 3}$. This outcome of the probe trials is again uninformative because of the failure to obtain a significant contrast effect.

Figure 3 shows the results from the second phase of training in which the sequence of components was changed to one in which the two target components were followed, rather than preceded, by the VI 30-sec versus extinction schedules. Initially, response rate was slightly higher in the target preceding the VI component, but this pattern gradually reversed over training, such that the higher rate occurred in the target component preceding extinction. The development of the anticipatory contrast effect was unusually slow (compare the reversal phase of Experiment 1, shown in Figure 1), which suggests that something about the prior training in Phase 1 may have retarded its development. In any event, anticipatory contrast was evident for all 4 subjects by the end of training. The results shown in Figure 3 were tested statistically with a twoway analysis of variance (ANOVA): blocks of sessions $x$ following schedule. Neither main effect was significant (both $F \mathrm{~S}<1.0$ ); however, the interaction was significant $[F(5,15)=2.92, p<.05]$. A test of simple effects showed that the effect of the following schedule was significant on the last block of training but not significant on any of the preceding blocks.

Figure 4 shows the results of the preference tests from the interspersed probe sessions. For all probe tests, regardless of the stage of training, there was a strong preference for the stimulus that was followed by the higher valued VI, consistent for all 4 subjects, and no consistent change across training. The results shown in Figure 4 were tested with a two-way ANOVA (session $\times$ following schedule). The main effect of the following schedule was significant $[F(1,3)=11.18, p<.05]$, but the effect of blocks $(F<1.0)$ and the interaction were not sig-

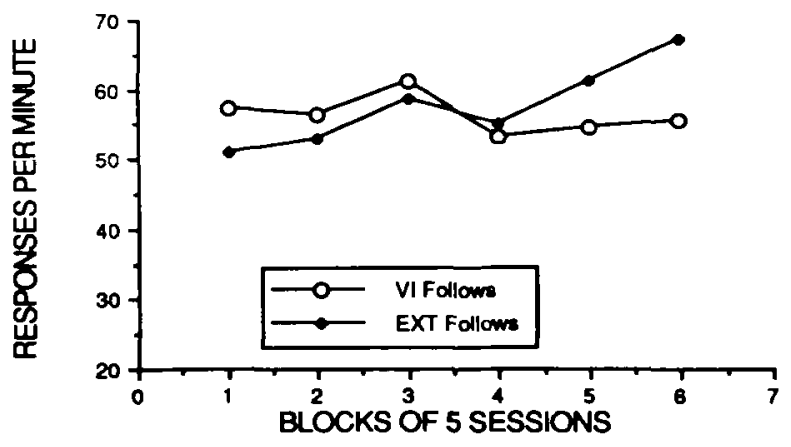

Figure 3. Response rates from the condition of Experiment 2 in which the two target components differed in terms of their following schedules. 


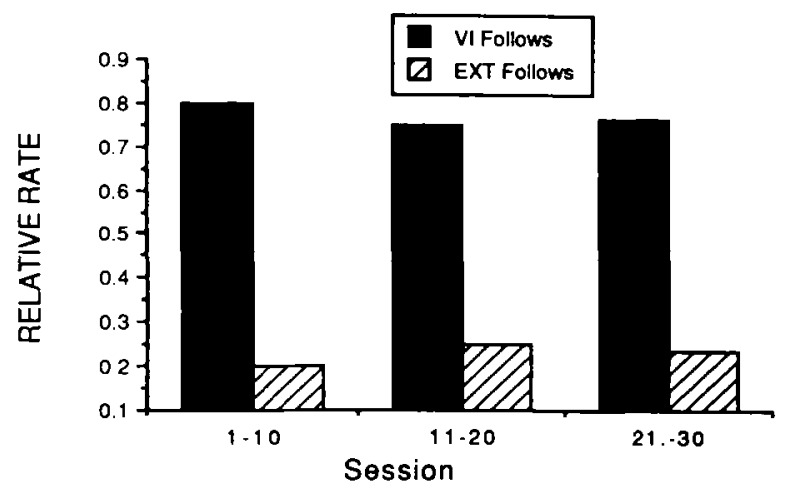

Figure 4. Relative response rates from the probe sessions of the condition of Experiment 2 in which the two target components differed in terms of their following schedules. Each bar shows the results of two probe sessions.

nificant $[F(2,6)=2.50, p>.10]$. Note that the lack of change does not correspond to the gradual development of anticipatory contrast that is seen in Figure 3. Although the anticipatory contrast effect with respect to response rate increased over training, preference for the VI $30-\mathrm{sec}$ schedule during the probe tests was virtually unchanged (mean preference during Tests 1 and 2 was 0.79 , and that during Tests 5 and 6 was 0.77 ).

\section{Discussion}

The results were consistent with those obtained in Experiment 1. Again, there was no reliable contrast effect due to different preceding schedules, and there was no consistent preference for the target stimuli following the different preceding schedules. Reliable contrast did $o c$ cur as a function of the different following schedules; again, there was a strong preference for the target stimulus followed by the higher valued schedule, showing that preference and contrast were inversely related.

The major new finding in Experiment 2 was that there was no correspondence between the development of contrast and the development of preference. Preference for the stimulus followed by the higher valued schedule occurred after only 5 sessions of training, whereas the development of anticipatory contrast did not occur until much later in training. This lack of correlation suggests that the two effects are under the control of different mechanisms that have different time courses.

One explanation of the lack of correspondence between the development of contrast and the development of preference is provided by Nevin et al. (1987), who have argued that it is essential to distinguish between Pavlovian and operant contingencies and that stimulus value is determined primarily by the former and response rate is determined by the latter. Thus, the relative predictiveness of a given keylight for food, including reinforcement during the keylight and following its presentation, determined the outcome of the preference tests, and the relative rate of reinforcement for the operant response during a par- ticular schedule component determined its response rate. Such an explanation implies that these two effects are independent and may summate or mutually interfere, depending upon the nature of the training situation. Although such a distinction is consistent with the present pattern of results, it is challenged by the nature of the contrast effect on response rate. In this and other studies (see especially Williams, 1981, and Williams \& Wixted, 1986), we have consistently found that contrast effects in steadystate procedures are due primarily to the following schedule of reinforcement, and that any residual effect due either to the preceding schedule or to the overall relative rate of reinforcement is either small in magnitude or inconsistent across subjects. The puzzle, therefore, is why contrast effects, which presumably depend upon the relative rate of reinforcement, occur reliably only when the Pavlovian signaling contingency with respect to the component stimuli is in the opposite direction of the relative rate of reinforcement. The stimulus-reinforcer contingencies should have at least some effect on response rate because of autoshaping effects, which is confirmed by other data (Brown et al., 1982; Williams, 1990a). Such effects should counteract any contrast effect due simply to the relative rate of reinforcement and thus reduce the size of the contrast effect that is evident. By comparison, with different preceding schedules, no such autoshaping effects should occur, so that the effects of overall relative rate of reinforcement on contrast should be undiminished. Thus, greater contrast effects should have occurred with different preceding schedules rather than with different following schedules, a prediction contrary to the present results and those of several previous studies (Williams, 1979, 1981; Williams \& Wixted, 1986). The implication is that the distinction between Pavlovian stimulus effects and "operant" effects of relative rate of reinforcement can be sustained only if some rationale can be offered for why the latter is primarily anticipatory in nature.

An alternative account of the present results is that preference may simply be a more sensitive measure than response rate of the value of the component and, thus, may be evident much earlier in training. To the extent that the effects on preference are due to the same processes as those on response rate, the implication is that increases in response rate do not necessarily reflect the value of the prevailing reinforcement schedule but may occur for other reasons. One possibility is emotionality effects, such as those proposed by frustration theory (Amsel, 1962); accordingly, response rate may simply reflect increases in arousal. Thus, the higher response rate preceding a period of extinction may indicate "anticipated frustration" in the same sense that response rate during the CS of a conditioned-suppression procedure may reflect "anticipated pain." Such an explanation has been offered previously by Leitenberg (1966) to explain the increase in response rate that is seen during a CS signaling periods of time-out from reinforcement. The difficulty with this explanation is that previous tests of frustration theory with free-operant contrast procedures have rejected it on the 
grounds that there is no relation between the amount of nonreinforced responding in the following component and the amount of responding in the target component (e.g., Williams, 1976, for a review, see Williams, 1983). It is possible, however, that the actual amount of nonreinforced responding is not the critical variable, because frustration may be due simply to changes in the availability of reinforcement, independent of the response contingencies.

A second possible explanation for the dissociation of response rate from stimulus value is that the contrast effects are due to orienting responses. Collins and Pearce (1985) have made a strong case that rate of keypecking in pigeons is due to two separate factors: (1) the strength of the association between the keylight and food, and (2) the amount of orienting behavior to the key as a function of the predictive accuracy of the key for subsequent events. More frequent orienting responses, and, thus, higher rates of pecking, occur when the keylight fails to predict the following effects accurately. Pearce and Collins (1987) have also shown that the response rates caused by different types of partial-reinforcement schedules do not predict the preference relations for different keylight stimuli correlated with those schedules. Instead, preference was determined by the rate of reinforcement associated with the keylight, independent of response rate, when the stimulus was presented alone. In other words, they produced dissociations between response rate and preference similar to those seen in the present experiments. But this similarity may be only superficial, in that their theory of orienting responses does not easily apply to the present procedures. Here, the two target components were equally predictive of their respective following schedules, and they differed only in terms of the nature of the events that were predicted. It is, nevertheless, possible that orienting responses play an important role in anticipatory contrast procedures, but for reasons not yet specified. If so, the implication would be that the present pattern of results would be unique to pigeons' keypecking in which the orienting response and the operant response are directed to the same location. In support of this possibility is the finding that rats do not show anticipatory contrast in procedures similar to those used here with pigeons (Williams, 1990b).

Whatever the explanation of the present dissociation between response rate and stimulus preference, this finding may occur in consummatory contrast procedures as well. Flaherty and his colleagues (Flaherty \& Checke, 1982; Flaherty \& Grigson, 1988; Flaherty \& Rowan, 1985) have shown that licking of a saccharin solution is suppressed if the opportunity to lick is followed by access to a higher valued sucrose solution. Greater suppression occurred the larger the disparity in value and the smaller the temporal separation between the two solutions. Such effects are similar to the effects on response rate observed here with free-operant procedures. However, they are contrary to findings from similar procedures that have studied conditioned taste preferences. For example, Holman (1975, Experiment 3) presented rats with either of two flavors in a weak saccharin solution, followed by either a strong saccharin solution or nothing. The results were that the flavor followed by the preferred saccharin solution was enhanced in value, as subjects drank more of it than the alternative when given a preference test. Thus, licking rates in Flaherty's experiments reflected a contrast effect, whereas preference tests in Holman's experiment reflected an increase in stimulus value. However, others have not always obtained this inverse relation (cf. Capaldi, Campbell, Sheffer, \& Bradford, 1987a, 1987b; Capaldi \& Sheffer, in press; Capaldi, Sheffer, \& Pulley, 1989). The critical procedural differences producing the different outcomes are not well defined, so that the determinants of the relation between the response rate and choice measures remain to be understood.

The present results have important theoretical implications apart from their bearing on theories of contrast. The dissociation of preference and response rate observed here fundamentally challenges theories of free-operant behavior that interpret response-rate effects in terms of a choice analysis. Herrnstein's (1970) analysis of singleresponse situations in terms of the matching law is the most salient example of such an approach, in that he argues that response rate in single-response situations is determined by the choice allocation between the behavior producing the experimenter-controlled reinforcers and the behaviors producing other uncontrolled reinforcers in the situations (e.g., scratching, preening, exploration). According to this analysis, response rate and choice are necessarily isomorphic except when differences in response rate change because of differences in the units in which responding is counted (e.g., VI and VR response rates differ because of incommensurate units, not because of differences in "response strength"'). However, there is no obvious way that differences in response units can account for the different outcomes seen here between measures of preference and measures of response rate when the choice alternatives were available successively (e.g., Figure 2). In the absence of such an account, the postulation that "all behavior is choice" seems seriously in question.

\section{REFERENCES}

AMSEL, A. (1962). Frustrative nonreward in partial reinforcement and discrimination learning. Psychological Review, 69, 306-328.

BLoomfield, T. M. (1969). Behavioural contrast and the peak shift In R. M. Gilbert \& N. S. Sutherland (Eds.), Animal discrimination leaming (Pp. 215-241). New York: Academic Press.

Brown, B. L., Hemmes, N. S., Coleman, D. A., Jr., Hassin, A., a Goldhammer, E. (1982). Specification of the stimulus-reinforcer relation in multiple schedules: Delay and probability of reinforcement Animal Learning \& Behavior, 10, 365-376.

Capaldi, E. D., Camprell, D. H., Sheffer, J. D., \& Bradford, J. P. (1987a). Conditioned flavour preferences based on delayed caloric consequences. Joumal of Experimental Psychology: Animal Behavior Processes, 13, 150-155.

Capald, E. D., Camprell, D. H., Sheffer, J. D., Bradford, J. P. (1987b). Nonreinforcing effects of giving "dessert" in rats. Appetite, 9, 99-112.

CaPaldi, E. D., ShefFer, J. D. (in press). Contrast and reinforcement in consumption. Learning \& Motivation. 
Capaldi, E. D., Sheffer, J. D., \& Pulley, R. J. (1989). Contrast effects in flavour preference learning. Quarterly Joumal of Experimental Psychology, 41B, 307-323.

Collins, L., Pearce, J. M. (1985). Predictive accuracy and the effects of partial reinforcement on serial autoshaping. Journal of Experimental Psychology: Animal Behavior Processes, 11, 548-564.

FlaherTy, C. F. (1982). Incentive contrast: A review of behavioral changes following shifts in reward. Animal Leaming \& Behavior, 10, 409-440.

Flaherty, C. F., Checke, S. (1982). Anticipation of incentive gain. Animal Learning \& Behavior, 10, 177-182.

Flaherty, C. F., \& Grigson, P. S. (1988). From contrast to reinforcement: Role of response contingency in anticipatory contrast. Journal of Experimental Psychology: Animal Behavior Processes, 14, 165-176.

FlamerTy, C. F., RowaN, G. A. (1985). Anticipatory contrast: Within-subjects analysis. Animal Learning \& Behavior, 13, 2-5.

Freeman, B. J. (1971). Behavioral contrast: Reinforcement frequency or response suppression? Psychological Bulletin, 75, 347-356.

HerRnstein, R. J. (1970). On the law of effect. Journal of the Experimental Analysis of Behavior, 13, 243-266.

Herrnstein, R. J., Heyman, G. M. (1979). Is matching compatible with reinforcement maximization on concurrent variable interval, variable ratio? Joumal of the Experimental Analysis of Behavior, 31, 209-223.

Holman, E. (1975). Immediate and delayed reinforcers for flavor preference in rats. Learning \& Motivation, 6, 91-100.

LEITENBERG, H. (1966). Conditioned acceleration and conditioned suppression in pigeons. Journal of the Experimental Analysis of Behavior, 9, 205-212.

Nevin, J. A. (1979). Reinforcement schedules and response strength. In M. D. Zeiler \& P. Harzem (Eds.), Advances in analysis of be haviour: Vol. 1. Reinforcement and the organization of behaviour (pp. 117-158). Chichester, England: Wiley.

Nevin, J. A., SMIth, L. D., \&oberTS, J. (1987). Does contingent reinforcement strength operant behavior? Journal of the Experimental Analysis of Behavior, 48, 17-33.
Pearce, J. M., * Coluns, L. C. (1987). An evaluation of the associative strength of a partially reinforced serial CS. Quarterly Journal of Experimental Psychology, 39B, 273-293.

Picker, M., Poling, A. (1982). Choice as a dependent measure in autoshaping: Sensitivity to frequency and duration of food presentation. Journal of the Experimental Analysis of Behavior, 37, 393-406.

REYNOLDS, G. S. (1961). Behavioral contrast. Joumal of the Experimental Analysis of Behavior, 4, 57-71.

TотА, M. E. (1990, May). Resistance to change in multiple schedules: Pavlovian influences. Paper presented at the meeting of the Association of Behavior Analysis, Nashville, TN.

Williams, B. A. (1976). Behavioral contrast as a function of the temporal location of reinforcement. Journal of the Experimental Analysis of Behavior, 26, 57-64.

Williams, B. A. (1979). Contrast, component duration, and the following schedule of reinforcement. Joumal of Experimental Psychology: Animal Behavior Processes, 5, 379-396.

WiLliams, B. A. (1981). The following schedule of reinforcement as a fundamental determinant of steady state contrast in multiple schedules. Journal of the Experimental Analysis of Behavior, 35, 293-310.

Williams, B. A. (1983). Another look at contrast in multiple schedules. Jourmal of the Experimental Analysis of Behavior, 39, 345-384.

Williams, B. A. (1989). Component duration effects in multiple schedules. Animal Learning \& Behavior, 17, 223-233.

Williams, B. A. (1990a). Pavlovian contingencies and anticipatory contrast. Animal Learning \& Behavior, 18, 44-50.

Williams, B. A. (1990b). Absence of anticipatory contrast in rats trained on multiple schedules. Joumal of the Experimental Analysis of Behavior, 53, 395-407.

WILLIAMS, B. A., \& WIXTED, J. T. (1986). An equation for behavioral contrast. Journal of the Experimental Analysis of Behavior, 45, 47-62.

(Manuscript received January 10, 1991; revision accepted for publication May 30, 1991.) 